\title{
Research Article \\ On the Application of the Fourier Series Solution to the Hydromagnetic Buoyant Two-Dimensional Laminar Vertical Jet
}

\author{
Marco Rosales-Vera, ${ }^{1,2}$ Yarko Niño, ${ }^{3}$ and Alvaro Valencia ${ }^{4}$ \\ ${ }^{1}$ High Temperatures Department, Instituto de Innovacion en Minería y Metalurgia, \\ Avenida del Valle 738, Santiago, Chile \\ ${ }^{2}$ Mechanical Engineering Department, Universidad de Chile, Beauchef 850, Santiago 8370448, Chile \\ ${ }^{3}$ Civil Engineering Department, Advanced Mining Technology Center, Universidad de Chile, \\ Blanco Encalada 2002, Santiago 8370449, Chile \\ ${ }^{4}$ Mechanical Engineering Department, Universidad de Chile, Beauchef 850, Santiago, Chile \\ Correspondence should be addressed to Alvaro Valencia, alvalenc@ing.uchile.cl
}

Received 9 August 2011; Accepted 21 October 2011

Academic Editor: Christos H. Skiadas

Copyright (C) 2012 Marco Rosales-Vera et al. This is an open access article distributed under the Creative Commons Attribution License, which permits unrestricted use, distribution, and reproduction in any medium, provided the original work is properly cited.

The problem of a hydromagnetic hot two-dimensional laminar jet issuing vertically into an otherwise quiescent fluid of a lower temperature is studied. We propose solutions to the boundary layer equations using the classical Fourier series. The method is essentiall to transform the boundary layer equations to a coupled set of nonlinear first-order ordinary differential equations through the Fourier series. The accuracy of the results has been tested by the comparison of the velocity distributions obtained by the Fourier series with those calculated by finite difference method. The results show that the present method, based on the Fourier series, is an efficient method, suitable to solve boundary layer equations applied to plane jet flows with high accuracy.

\section{Introduction}

The behavior of a two-dimensional symmetric plane jet that spreads out from a nozzle into a fluid at rest is of basic fluid dynamics interest with many applications in engineering.

Several authors have applied the boundary layer equations to jet flows with very good results $[1,2]$. The exact analytical solutions found by these authors consider that the size of the nozzle is infinitely small. Crane extends the problem to jets in an atmosphere 
of different densities [3, 4]. Pozzi and Bassano [5] introduced the Padé approximants to the study of plane jets. Revuelta et al. [6] discussed the idea of the virtual origin of the jet as a first-order correction for the far-field description, with the idea of obtaining better agreement between the observed flow field and the one predicted in $[1,2]$ when the size of the nozzle is finite. More recently, Wilks et al. studied the development of a jet into uniform and nonuniform external stream [7-9]. Brand and Lahey [10] studied the effect of the buoyancy in axisymmetric and plane jets using the self-similar solution of the boundary layer equations. Sánchez-Sanz et al. [11] studied the hydrogen laminar jet using asymptotic methods. Martynenko et al. [12] presented an analysis of the buoyancy effects on momentum and heat transfer in vertical jets and plumes. The works mentioned above are based on self-similar solutions of the boundary layer equations. The self-similar solutions allow for the transformation of the boundary layer equations into ordinary differential equations [8]. When the width of the nozzle is finite, generally it is not possible to find self-similar solutions to the boundary layer equations; in this case it must resort to numerical solutions [13-15]. In $1992 \mathrm{Yu}$ et al. [16] presented a rigorous numerical solution for free and wall two-dimensional buoyant jets. More recently, Aissia et al. [17] have performed a numerical analysis for the aiding buoyancy on a vertical round jet. In 2007 Ali et al. [18] gave a detailed numerical study of the influence of the Prandtl number on the hydrodynamic and thermal behaviour of a laminar buoyant free jet. Recently, the plane jet problem was revisited by Rosales-Vera and Valencia [19].

Concerning the application of spectral methods to boundary layer flow problems, Kumar and Yajnik [20] studied a channel flow problem including a sudden expansion, using an expansion in the eigenfunctions of the Poiseuille flow development, and the problem is reduced to solve nonlinear first-order ordinary differential equations that have a tendency to decouple rapidly. In 1982 Plotkin [21] solved the same problem, specifically by applying the Fourier series, getting solutions for a sudden channel expansion, channel entry flow, and slowly diverging cannel, which agree well with the results of previous investigations. More recently, Figueira Da Silva and Cotta [22] solved the entrance channel flow problem by applying integral transform techniques to the boundary layer equations. Khabibrakhmanov and Summers [23] resolved the Blasius equation, using a spectral method with generalized Laguerre polynomials.

In 2004, Rosales and Frederick [24] solved the classical Graetz problem by applying the Fourier series. This solution has the virtue of delivering very precise numerical results for the Nusselt number in the area near the entrance to the channel. Ma et al. [25] develop a Hermite spectral method for parabolic equations in unbounded domains. Recently, Rosales and Valencia [26] studied the Blasius equation by the Fourier series finding a solution with very high accuracy. The same authors applied the Fourier series to the Falkner-Skan equation [27]. The results show that the pseudospectral method has a very high accuracy. A complete review of spectral methods applied to fluid dynamics can be found in [28]. About the pseudospectral methods applied to jet flow, Rosales-Vera and Valencia [19] applied the Fourier series to study the fluid dynamic of the free plane jet.

In this paper, a spectral method using the Fourier series expansions is used to solve the buoyant two-dimensional laminar vertical jet of a conducting fluid in a magnetic field (hydromagnetic jet) with the width of the nozzle being finite. The method is essentiall, through the Fourier series, to transform the boundary layer equations to a coupled set of nonlinear first-order ordinary differential equations. 


\section{Analysis}

Consider the steady, laminar, hydromagnetic flow combined with heat transfer by natural convection of a vertical jet flow, where a magnetic field of uniform strength $B_{0}$ is applied in the $Y$ direction which is normal to the $X$ axis of the jet direction.

In this case the momentum and energy equations of the hydromagnetic vertical buoyant plane jet flow derived from the Boundary layer approximation are

$$
\begin{gathered}
u \frac{\partial u}{\partial x}+v \frac{\partial u}{\partial y}=\frac{\partial^{2} u}{\partial y^{2}}+\frac{G r}{\operatorname{Re}} \vartheta-M^{2} u \\
\frac{\partial u}{\partial x}+\frac{\partial v}{\partial y}=0 \\
u \frac{\partial \vartheta}{\partial x}+v \frac{\partial \vartheta}{\partial y}=\frac{1}{\operatorname{Pr}} \frac{\partial^{2} \vartheta}{\partial y^{2}}
\end{gathered}
$$

where the dimensionless coordinates are

$$
y=\frac{Y}{a}, \quad x=\frac{X}{\operatorname{Re} a}, \quad u=\frac{U}{U_{0}}, \quad v=\operatorname{Re} \frac{V}{U_{0}}, \quad \vartheta(\eta)=\frac{T-T_{\infty}}{T_{0}-T_{\infty}},
$$

where $a$ is the width of the nozzle, $U_{0}$ is the velocity flow in the nozzle, $U$ and $V$ are the velocities in the $X$ and $Y$ directions, $T$ is the temperature flow, and $T_{0}$ and $T_{\infty}$ are the temperature flow in the nozzle and the temperature of the fluid far of the jet, respectively. The Reynolds number is given by $\operatorname{Re}=\rho U_{0} a / \mu, \rho$ and $\mu$ are the density and the viscosity of the fluid, $M=B_{0} a \sqrt{\sigma} / \sqrt{\mu}$ is the Hartmann number, $B_{0}$ is the magnetic field, $\sigma$ is the conductivity of the fluid, $\mathrm{Gr}=\left(\rho^{2} / \mu^{2}\right) g \beta\left(T_{0}-T_{\infty}\right) a^{3}$ is the Grashof number, $\mathrm{Ri}=\mathrm{Gr} / \mathrm{Re}^{2}$ is the Richardson number, Pr is the Prandtl number, and $\beta$ is the thermal diffusivity. The boundary conditions are

$$
\begin{array}{ll}
u(0, y)=u_{0}(y), \quad u(x, y \longrightarrow \infty)=0,\left.\quad \frac{\partial u}{\partial y}\right|_{y=0}=0, \\
\vartheta(0, y)=\vartheta_{0}(y), \quad \vartheta(x, y \longrightarrow \infty)=0,\left.\quad \frac{\partial \vartheta}{\partial y}\right|_{y=0}=0 .
\end{array}
$$

The following solution for the velocity field and temperature is considered:

$$
\begin{gathered}
u(x, y)=\sum_{i=1}^{\infty} f_{i}(x) \cos \left(k_{i} y\right), \\
v(x, y)=-\sum_{i=1}^{\infty} \frac{1}{k_{i}} f_{i}^{\prime}(x) \sin \left(k_{i} y\right), \\
\vartheta(x, y)=\sum_{i=1}^{\infty} g_{i}(x) \cos \left(k_{i} y\right),
\end{gathered}
$$

where the number of wavelength $k_{i}$ is given by $k_{i}=(\pi / 2 L)(2 i-1)$. 
The $y$ dependence is built on cosine functions that automatically satisfy the boundary conditions at the $y=L$ and at the symmetry plane for $u$. These functions form an orthonormal basis, as follows:

$$
\int_{0}^{L} \cos \left(k_{i} y\right) \cos \left(k_{j} y\right) d y=\frac{L}{2} \delta_{i j}
$$

Replacing (2.6) in (2.1),

$$
\begin{aligned}
& \sum_{i, j=1}^{\infty} f_{i}(x) f_{j}^{\prime}(x) \cos \left(k_{i} y\right) \cos \left(k_{j} y\right)+\sum_{i, j=1}^{\infty} \frac{k_{j}}{k_{i}} f_{i}^{\prime}(x) f_{j}(x) \sin \left(k_{i} y\right) \sin \left(k_{j} y\right) \\
& =-\sum_{i=1}^{\infty} k_{i}^{2} f_{i}(x) \cos \left(k_{i} y\right)+\frac{\mathrm{Gr}}{\operatorname{Re}} \sum_{i=1}^{\infty} g_{i}(x) \cos \left(k_{i} y\right)-M^{2} \sum_{i=1}^{\infty} f_{i}(x) \cos \left(k_{i} y\right)
\end{aligned}
$$

Multiplying (2.8) by $\cos \left(k_{p} y\right)$ and integrating from $y=0$ to $L$, the following system of equations is obtained:

$$
\begin{gathered}
\frac{1}{2} \sum_{i, j=1}^{\infty}\left(f_{i}(x) f_{j}^{\prime}(x)-\frac{k_{j}}{k_{i}} f_{i}^{\prime}(x) f_{j}(x)\right) I_{i j}^{p}+\frac{1}{2} \sum_{i, j=1}^{\infty}\left(f_{i}(x) f_{j}^{\prime}(x)+\frac{k_{j}}{k_{i}} f_{i}^{\prime}(x) f_{j}(x)\right) J_{i, j}^{p} \\
=-\frac{L}{2} \sum_{i=1}^{\infty} k_{i}^{2} f_{i}(x) \delta_{i p}+\frac{L}{2} \frac{\mathrm{Gr}}{\operatorname{Re}} g_{i}(x) \delta_{i p}-M^{2} \frac{L}{2} \sum_{i=1}^{\infty} f_{i}(x) \delta_{i p},
\end{gathered}
$$

where

$$
I_{i j}^{p}=(-1)^{i+j+p+1} \frac{k_{p}}{\left(k_{i}+k_{j}\right)^{2}-k_{p}^{2}}, \quad J_{i j}^{p}(-1)^{i+j+p} \frac{k_{p}}{\left(k_{i}-k_{j}\right)^{2}-k_{p}^{2}}
$$

The following set of equations $(p=1, \ldots, N)$ is gotten, where $N$ is the number of equations used to approximate the solution with a finite number of terms in the Fourier series. Equation (2.9) can be expressed as follows:

$$
\frac{1}{2} \sum_{i, j=1}^{N} f_{i}\left(A_{i j}^{p}+B_{i j}^{p}+D_{i j}^{p}-C_{i j}^{p}\right) f_{i}^{\prime}=-\frac{L}{2} k_{p}^{2} f_{p}(x)+\frac{L}{2} \frac{\mathrm{Gr}}{\operatorname{Re}} g_{p}(x)-M^{2} \frac{L}{2} f_{p}(x) \quad(p=1, \ldots, N),
$$

where

$$
A_{i j}^{p}=I_{i j}^{p}, \quad B_{i j}^{p}=J_{i j}^{p}, \quad C_{i j}^{p}=I_{i j}^{p} \frac{k_{i}}{k_{j}}, \quad D_{i j}^{p}=J_{i j}^{p} \frac{k_{i}}{k_{j}} .
$$


Doing a similar analysis to (2.3), the following set of equations is obtained:

$$
\frac{1}{2} \sum_{i, j=1}^{N} f_{i}\left(A_{i j}^{p}+B_{i j}^{p}\right) g_{j}^{\prime}+\frac{1}{2} \sum_{i, j=1}^{N} g_{i}\left(D_{i j}^{p}-C_{i j}^{p}\right) f_{i}^{\prime}=-\frac{1}{\operatorname{Pr}} \frac{L}{2} k_{p}^{2} g_{p}(x) \quad(p=1, \ldots, N) .
$$

Equations (2.11) and (2.13) represent a first-order nonlinear system of equations. The functions $f$ and $g$, which account for the axial dependence of velocity field and temperature field, can be determined as follows.

\section{Numerical Solution}

Equations (2.11) and (2.13) are discretized in the variable $x$, being the following system of algebraic equations:

$$
\begin{gathered}
\sum_{i, j=1}^{N} f_{i}(x) M_{i j}^{p}\left(f_{j}(x)-f_{j}(x-\Delta x)\right)+\left(k_{p}^{2} f_{p}(x)-\frac{\mathrm{Gr}}{\operatorname{Re}} g_{p}(x)+M^{2} f_{p}(x)\right) L \Delta x=0, \\
\sum_{i, j=1}^{N} f_{i} F_{i j}^{p}\left(g_{j}(x)-g_{j}(x-\Delta x)\right)+\sum_{i, j=1}^{N} g_{i} H_{i j}^{p}\left(f_{j}(x)-f_{j}(x-\Delta x)\right)+\frac{L}{\operatorname{Pr}} k_{p}^{2} g_{p}(x) \Delta x=0 .
\end{gathered}
$$

The numerical solution of the systems (2.11) and (2.12) is for each station $x$. To solve the system of algebraic equations for the coefficients $f_{p}(x)$ and $g_{p}(x)$, where the values of the coefficients $f_{p}(x-\Delta x)$ and $g_{p}(x-\Delta x)$ are known from the station $(x-\Delta x)$, the solution is found through an iterative method for coefficients of the Fourier series, defining the following vectors:

$$
\begin{aligned}
G_{p}^{(1)}(\vec{f}(x), \vec{g}(x))= & \sum_{i, j=1}^{N} f_{i}(x) M_{i j}^{p}\left(f_{j}(x)-f_{j}(x-\Delta x)\right) \\
& +\left(k_{p}^{2} f_{p}(x)-\frac{\mathrm{Gr}}{\operatorname{Re}} g_{p}(x)+M^{2} f_{p}(x)\right) L \Delta x, \\
G_{p}^{(2)}(\vec{f}(x), \vec{g}(x))= & \sum_{i, j=1}^{N} f_{i} F_{i j}^{p}\left(g_{j}(x)-g_{j}(x-\Delta x)\right) \\
& +\sum_{i, j=1}^{N} g_{i} H_{i j}^{p}\left(f_{j}(x)-f_{j}(x-\Delta x)\right)+\frac{L}{\operatorname{Pr}} k_{p}^{2} g_{p}(x) \Delta x .
\end{aligned}
$$

So, the functions $f_{i}(x)$ and $g_{i}(x)$ of the Fourier series may be recursively determined by the following equations:

$$
\begin{aligned}
& \vec{f}^{(n+1)}=\vec{f}^{(n)}-\left[J^{(1)}\left(\vec{f}^{(n)}\right)\right]^{-1} \vec{G}^{(1)}\left(\vec{f}^{(n)}, \vec{g}^{(n)}\right), \\
& \vec{g}^{(n+1)}=\vec{g}^{(n)}-\left[J^{(2)}\left(\vec{g}^{(n)}\right)\right]^{-1} \vec{G}^{(n)}\left(\vec{f}^{(n)}, \vec{g}^{(n)}\right),
\end{aligned}
$$


where the Jacobian matrixes are given by

$$
\begin{gathered}
J_{p j}^{(1)}=\frac{\partial G_{p}^{(1)}}{\partial f_{j}}=\sum_{i=1}^{N} f_{i}(x) M_{i j}^{p}+\sum_{i=1}^{N} M_{j i}^{p}\left(f_{i}(x)-f_{i}(x-\Delta x)\right)+\left(k_{p}^{2}+M^{2}\right) L \Delta x \delta_{p j}, \\
J_{p j}^{(2)}=\frac{\partial G_{p}^{(2)}}{\partial g_{j}}=\sum_{i=1}^{N} f_{i} F_{i j}^{p}+\sum_{i=1}^{N} H_{j i}^{p}\left(f_{i}(x)-f_{i}(x-\Delta x)\right)+\frac{L}{\operatorname{Pr}} k_{p}^{2} \Delta x \delta_{p j},
\end{gathered}
$$

and the vectors $\vec{f}^{(n)}, \vec{g}^{(n)}$ represent the values of $\vec{f}, \vec{g}$ in the $n$ iteration. The iterations for $\vec{f}^{(n)}, \vec{g}^{(n)}$ from (2.11) may be repeated until $\left\|\vec{a}^{(n+1)}-\vec{a}^{(n)}\right\| \leq \varepsilon$, for some prescribed error tolerance $\varepsilon$.

The boundary condition at $x=0$ is considered a uniform profile and a profile of parabolic shape.

In the uniform initial profile, one has

$$
u(0, y)=\left\{\begin{array}{ll}
1 & 0 \leq y \leq \frac{1}{2} \\
0 & \frac{1}{2}<y \leq L
\end{array} \quad \vartheta(0, y)= \begin{cases}1 & 0 \leq y \leq \frac{1}{2} \\
0 & \frac{1}{2}<y \leq L\end{cases}\right.
$$

In the space of Fourier, the vectors $f_{i}(x=0)$ and $g_{i}(x=0)$ are obtained from the previous condition:

$$
\begin{aligned}
& f_{i}(0)=\frac{2}{L} \int_{0}^{L} u(0, y) \cos \left(k_{i} y\right) d y=\frac{2}{L} \frac{\sin k_{i} / 2}{k_{i}}, \\
& g_{i}(0)=\frac{2}{L} \int_{0}^{L} \vartheta(0, y) \cos \left(k_{i} y\right) d y=\frac{2}{L} \frac{\sin k_{i} / 2}{k_{i}} .
\end{aligned}
$$

In the parabolic initial profile, one has

$$
u(0, y)=\left\{\begin{array}{ll}
\sqrt{\frac{15}{8}}\left(1-(2 y)^{2}\right), & 0 \leq y \leq \frac{1}{2}, \\
0, & \frac{1}{2}<y \leq L .
\end{array} \quad \vartheta(0, y)= \begin{cases}1, & 0 \leq y \leq \frac{1}{2} \\
0, & \frac{1}{2}<y \leq L .\end{cases}\right.
$$

In the space of Fourier, the vectors $f_{i}(x=0)$ and $g_{i}(x=0)$ are obtained from the previous condition:

$$
f_{i}(0)=\frac{2}{L} \int_{0}^{L} u(0, y) \cos \left(k_{i} y\right) d y=\frac{8}{L k_{i}} \sqrt{\frac{15}{2}}\left(\frac{\sin (1 / 2) k_{i}}{k_{i}^{2}}-\frac{1 / 2}{k_{i}} \cos \frac{1}{2} k_{i}\right)
$$




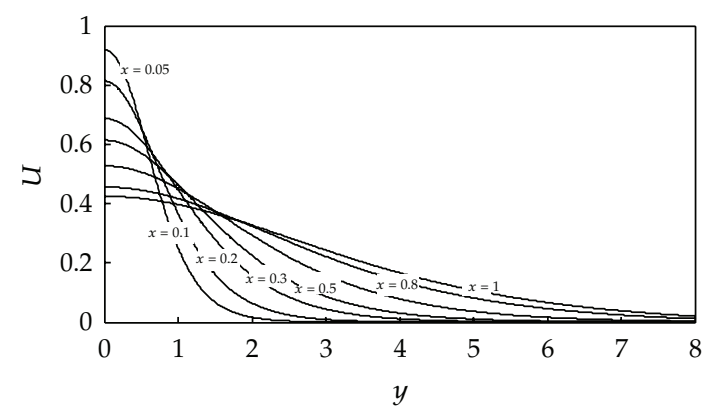

Figure 1: Velocity field for a free jet with a uniform initial velocity profile $(\operatorname{Pr}=1, \operatorname{Re}=1250)$.

\section{Results}

\subsection{Free Plane Jet}

The case of the free plane jet is obtained when $M=0, \operatorname{Ri}=0$, and $\operatorname{Pr} \neq 0$. Figure 1 shows the velocity field obtained for a uniform initial velocity profile with $\operatorname{Pr}=1$ and $\operatorname{Re}=1250$, in different $x$ position.

In this case, the velocity field is not coupled with the temperature field. In the far field, the velocity field is the similarity solution of Bickley [1], where the centerline velocity is given by $u_{c}(x)=C / x^{1 / 3}$, where $C=0.4543$ for a rectangular initial profile (Bickley [1, Figure 2]) with $\mathrm{Ri}=0$. The case of a parabolic initial profile using, Fourier series was reported by Rosales-Vera and Valencia [19].

\subsection{Buoyant Vertical Jet}

The case of a buoyant two-dimensional laminar vertical jet is obtained when $M=0, \mathrm{Ri} \neq 0$, and $\operatorname{Pr} \neq 0$. The Reynolds number was kept constant $(R e=1250)$ to validate the numerical results in [18]. Figure 2 shows the evolution of the centerline velocity profile as function of the $x$ distance from the nozzle exit for several Richardson numbers with a uniform initial velocity profile. The Richardson number varies from 0 to 0.2 , considering the forced convection case. This, in reality, with $\operatorname{Re}=1250$, considers $G r$ between 0 and 312500 for $\operatorname{Pr}=0.71$ (air). The centerline velocity $u$ distribution obtained from the Fourier series solution (solid line) is compared with the solution reported in [18] using finite differences (points). The results coincide very well.

For the field near to the exit of the nozzle, the jet velocity remains constant with a value equal to the initial velocity, which represents the length of the potential core. For Richardson number less than $\sim 10^{-3}$, the length of the potential core is $x \sim 10 \operatorname{Re} 2 h$. When Ri $>10^{-3}$, the length of the potential core is drastically reduced. In this case the effect of the buoyancy is present from distances $x \sim 0.1 \operatorname{Re} 2 h$ very near to the nozzle.

Figure 3 shows the evolution of the dimensionless centerline temperature profile as function of the $x$ distance from the nozzle exit, for several Richardson numbers. For the field near to the exit of the nozzle, the centerline temperature remains constant with a value equal to the initial temperature. This is the length of the potential core of the temperature field, and no dependence on the Richardson number exists. The length of this region is $x \sim 10 \operatorname{Re} 2 h$. 


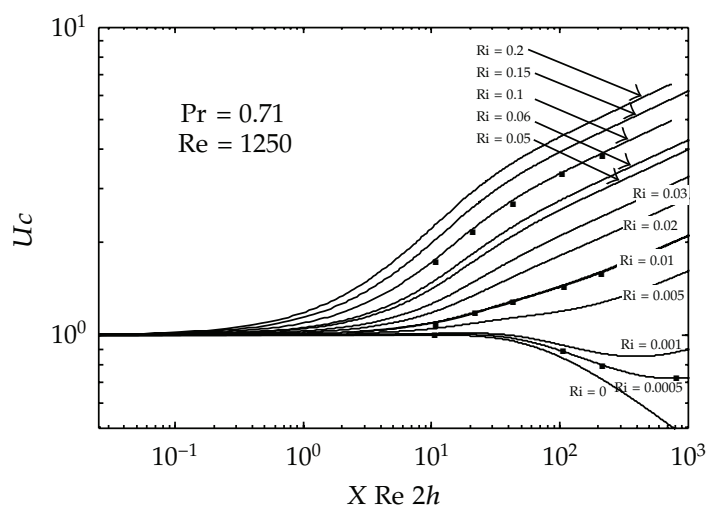

Figure 2: Centerline velocity profile as function of the distance from the nozzle exit for several Richardson numbers, with uniform initial velocity profile. The Fourier series (solid line), finite differences (points) $[18]$.

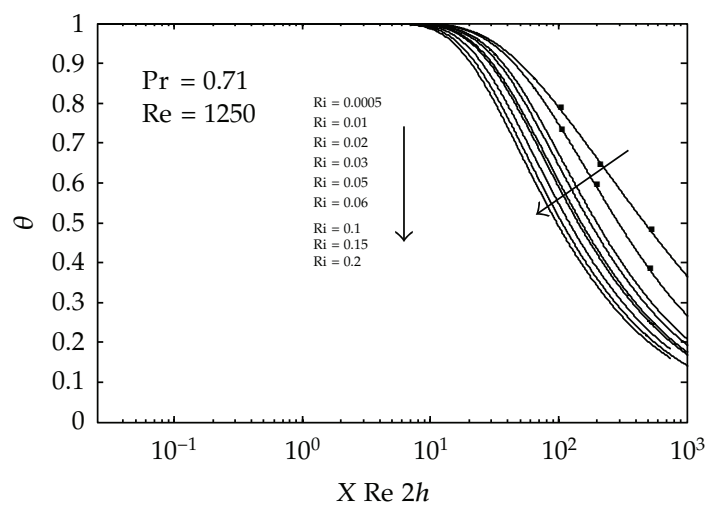

Figure 3: Centerline temperature profile as function of the $x$ distance from the nozzle exit, for several Richardson numbers. The Fourier series (solid line), finite differences (points) [18].

Figure 3 shows that the jet core temperature falls faster by increasing the Richardson number. This is because the central jet velocity increases with the increase in the Richardson number; thus, the heat exchange is more efficient.

The results and analysis of a jet for a parabolic initial velocity profile can be found in [18]. The general behaviour of the jet does not depend on the initial velocity profile.

\subsection{Hydromagnetic Buoyant Vertical Jet}

The case of a hydromagnetic buoyant two-dimensional laminar vertical jet is obtained when $M \neq 0, R i \neq 0$, and $\operatorname{Pr} \neq 0$. The case $\mathrm{Ri}=0$ was studied by Moreau [29], finding similarity solutions for the velocity field in the far field. A numerical study was conducted by Vlachopoulos [30].

Figure 4 shows the evolution of the centerline velocity profile as a function of the $x$ distance from the nozzle exit for $\mathrm{Ri}=0.01$ and several Hartmann numbers with a uniform initial velocity profile. The results show that in the near-field region, the centerline velocity 


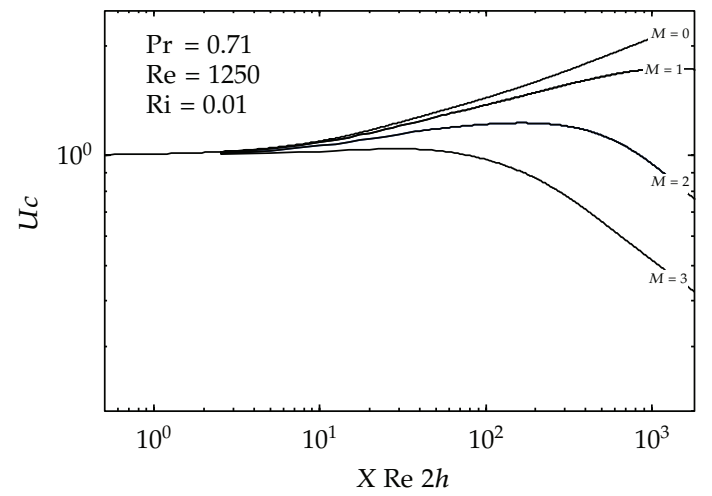

Figure 4: Centerline velocity profile as a function of the distance from the nozzle exit for several magnetic numbers with uniform initial velocity profile.

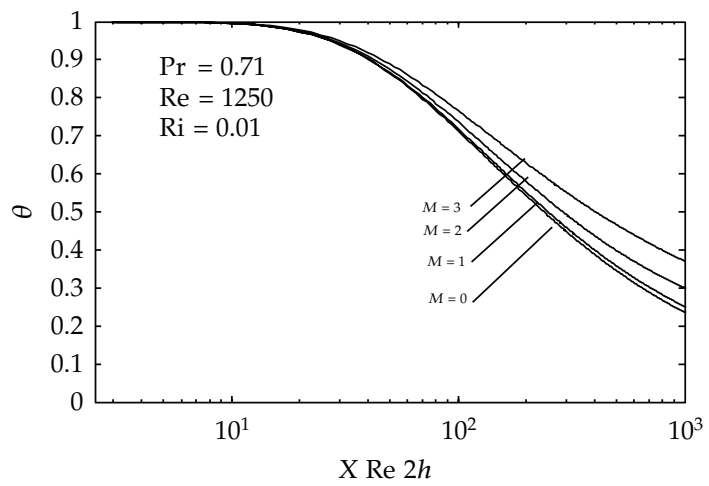

Figure 5: Centerline temperature as a function of the distance from the nozzle exit for several magnetic numbers with uniform initial velocity profile.

remains constant with a value equal to the initial velocity, as in the case of pure buoyant jet. However as $x$ is increased, the centerline velocity of the jet is considerably shortened by increasing the Hartmann number. The effect of the magnetic field is effective to start at $x \sim \operatorname{Re} 2 h$. The deceleration of the jet makes the decrease of temperature in the jet axis slower, weakening the heat transfer between the colder jet and the cold atmosphere (Figure 5). However the effect of the magnetic field on the temperature is less intense than in the velocity field.

Figure 6 shows the effect of the magnetic field in the velocity profile in $x=0.8$ (far field), for several values of Hartmann number. The magnetic field intensifies so the central velocity of the jet decreases making the velocity profile wide and less intense. Then, if the magnetic field continues to increase, it will reach the point where the buoyancy vanishes. This is because the Hartmann number reached a value such that $M^{2} \sim \mathrm{Gr} / \mathrm{Re}$; considering in particular the case $\operatorname{Pr} \sim 1,(2.1)-(2.3)$ with initial conditions (2.5) become the equations for a free jet with the solution $u(x, y)=\theta(x, y)$; then, in this case the magnetic field completely cancels the effect of buoyancy. This phenomenon is shown in Figure 6, where the dashed line represents the velocity profile for a free jet. The profile is very similar to the velocity profile obtained for $M=3$, where $M^{2} \sim \mathrm{Gr} /$ Re. 


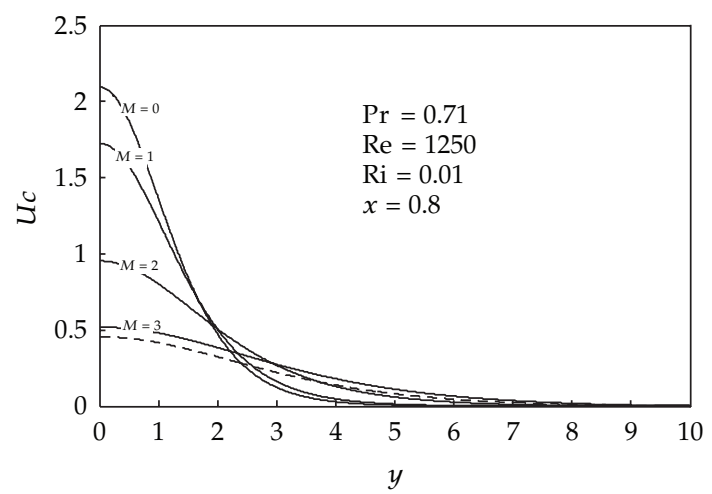

Figure 6: Effect of the magnetic field in the velocity profile in $x=0.8$ (far field), for several values of the Hartmann numbers with a uniform initial velocity profile. The dashed line represents the velocity profile for a free jet.

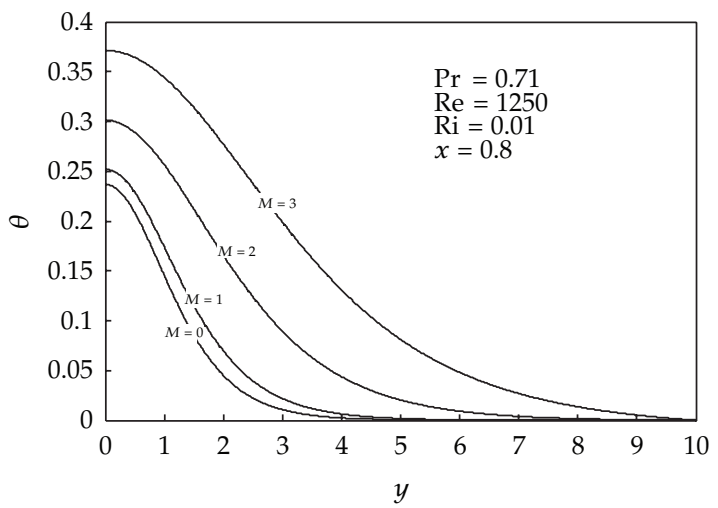

Figure 7: Effect of the magnetic field in the temperature profile in $x=0.8$ (far field), for several values of Hartmann numbers with a uniform initial velocity profile.

Figure 7 shows the effect of magnetic field on the temperature profiles for $x=0.8$. The temperature profile is widening, for a fixed distance $x$, to the extent that the magnetic field increases. The temperature in the jet is more homogeneous, and the core temperature, as mentioned above, will generate increasing jet cooling.

Results obtained in the previous case remain valid for other Richardson numbers. Figure 8 shows the evolution of the centerline velocity profile as function of the $x$ distance from the nozzle exit for $\mathrm{Ri}=0.1$ and 0.01 . In the figure, the continuous line represents a uniform initial velocity profile and the dashed line represents a parabolic initial profile. When increasing the Richardson number, the central velocity increases due to increased jet buoyancy. However, the centerline velocity is also reduced when the magnetic field is increased, reducing the effect of jet buoyancy. Concerning the influence of the initial velocity profile on the development of the central jet velocity, Figure 8 shows that there are differences only in areas very close to the inlet, since for relatively small distances $(x \sim 0.8)$ the central velocity acquires the asymptotic solution. Both cases, the uniform and the parabolic profile, converge to a solution of the far field where the initial profile has no influence. 


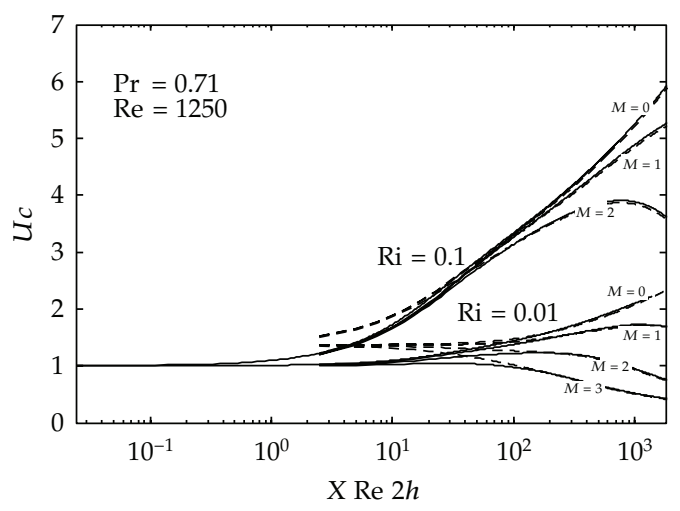

Figure 8: Centerline velocity profile as function of the distance from the nozzle exit for several Hartmann numbers and Richardson number 0.1 and 0.01 . Continuous line corresponds to uniform initial velocity profile, while dashed line corresponds to parabolic initial profile.

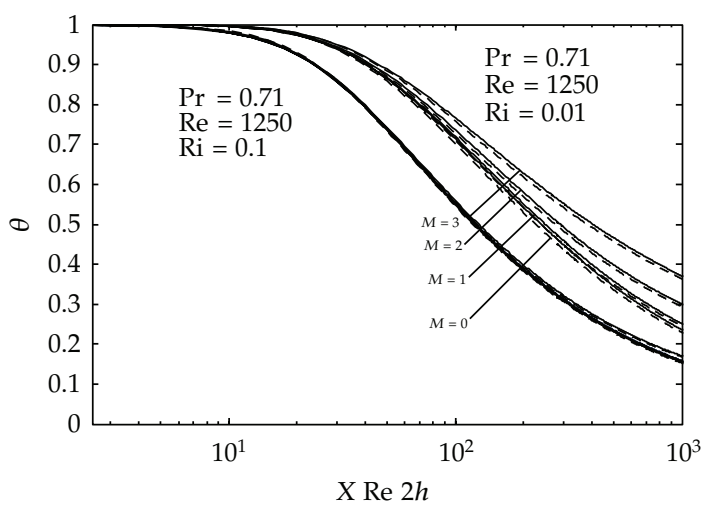

Figure 9: Centerline temperature profile as function of the distance from the nozzle exit for several Hartmann numbers and Richardson number 0.1 and 0.01 . Continuous line corresponds to uniform initial velocity profile, while dashed line corresponds to parabolic initial profile.

Figure 9 shows that the initial velocity profile has little effect on the centerline temperature, both in the near field and the far field. Regarding the influence of magnetic field, like in Figure 4, when the Hartmann number increases, the temperature tends to fall more slowly, slowing the cooling jet. This effect is reduced when the Richardson number is increased. For example, for $\mathrm{Ri}=0.1$, the effect of the magnetic field is much less important than in the case of $\mathrm{Ri}=0.01$, as in the case where the Richardson number is equal to 0.1, the axial temperature profile remains virtually unchanged with increasing the Hartmann number.

Figure 10 shows the evolution of the centerline velocity profile as function of the $x$ distance from the nozzle exit for $\mathrm{Ri}=0.1$ and 0.01. In both cases the Prandtl number was $\operatorname{Pr}=9$. The figure shows a similar behaviour to the case $\operatorname{Pr}=0.71$ (Figure 8 ). This shows that the Prandtl number has little effect on the center velocity of the jet. However, this is not true in the temperature field. Figure 11 shows the temperature profiles at $x=0.8$, for $\mathrm{Ri}=0.01$, and the Prandtl number values $\mathrm{Pr}=0.71$ and 9. As expected, the Prandtl number has a great influence on the temperature profiles. To increase the Prandtl number, the temperature profile 


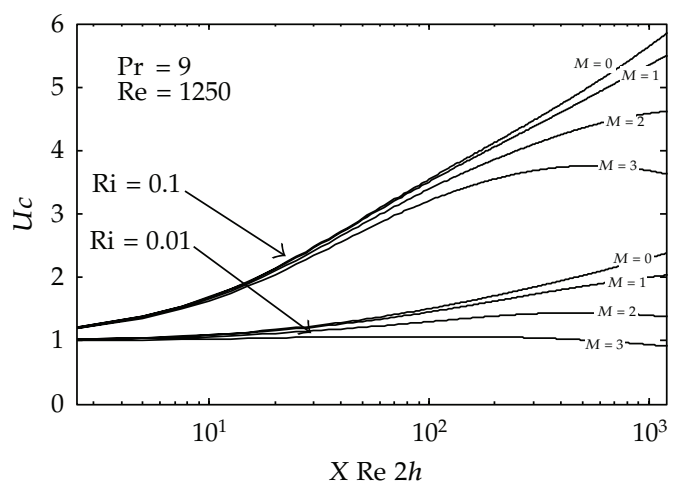

Figure 10: Centerline velocity profile as function of the distance from the nozzle exit for several Hartmann numbers, Richardson number 0.1 and 0.01 , and Prandtl number 9.

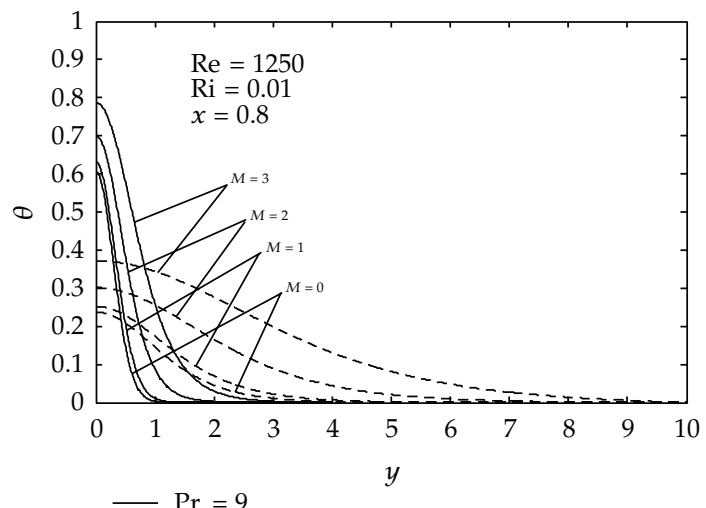

$$
--\operatorname{Pr}=0.71
$$

Figure 11: Effect of the magnetic field on the temperature profile in $x=0.8$ (far field), for several values of Hartmann numbers with a uniform initial velocity profile. and Prandtl number 0.71 and 9.

becomes narrow, so that the jet maintains its center temperature for longer distances, slowing the cooling of the jet. Thus the buoyancy stays longer and central jet velocity is increased slightly.

Figure 12 shows the evolution of the centerline temperature profile as function of the $x$ distance from the nozzle exit for $\mathrm{Ri}=0.01$ and 0.1 . The results show a similar result in Figure 9; when the Hartmann number increases, the temperature tends to fall more slowly, slowing the cooling jet. This effect is reduced when the Richardson number is increased. For example, for $\mathrm{Ri}=0.1$, the effect of the magnetic field is much less important than in the case of $\mathrm{Ri}=0.01$.

\section{Conclusion}

This paper demonstrates that the Fourier series is a suitable method to solve the boundary layer problem applied to jets. The hydromagnetic buoyant two-dimensional laminar vertical jet was analyzed. In particular, we studied the combined effect of the Hartmann number 


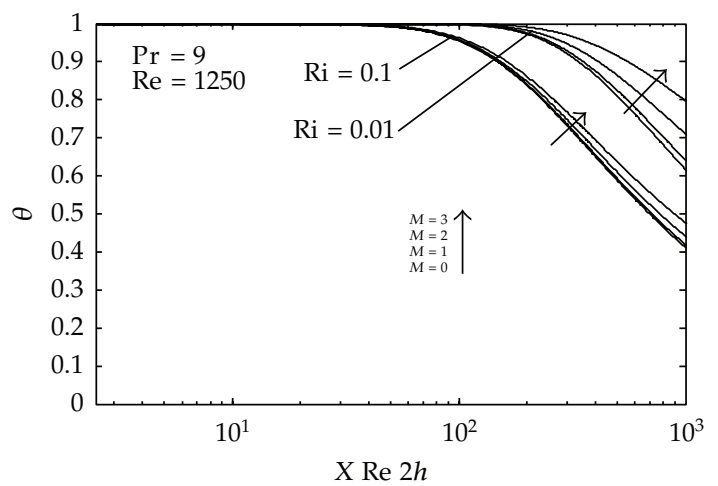

Figure 12: Centerline temperature profile as function of the distance from the nozzle exit for several Hartmann numbers, Richardson number 0.1 and 0.01 , and Prandtl number 9.

and the Prandtl number, showing that the magnetic field could vanish the effect of natural convection. The results obtained show a high accuracy. The present method can be easily extended to more complex flows, for example, jets with heat and mass transfer including multiphase flow and combustion problems.

\section{References}

[1] W. G. Bickley, “The plane jet," Philosophical Magazine, vol. 23, 1937.

[2] H. Schlichting, Boundary Layer Theory, McGraw-Hill, New York, NY, USA, 4th edition, 1955.

[3] L. J. Crane and D. C. Pack, "The mixing of a jet of gas with an atmosphere of a different gas at large distances from the orifice. I. The plane jet," The Quarterly Journal of Mechanics and Applied Mathematics, vol. 14, pp. 385-391, 1961.

[4] L. J. Crane, "The mixing of a jet of gas with an atmosphere of a different gas at large distances from the orifice. II. The round jet," The Quarterly Journal of Mechanics and Applied Mathematics, vol. 14, pp. 393-402, 1961.

[5] A. Pozzi and E. Bassano, "Application of Padé approximants to the study of plane jets," Computers $\mathcal{E}$ Mathematics with Applications, vol. 30, no. 11, pp. 107-123, 1995.

[6] A. Revuelta, A. L. Sánchez, and A. Liñán, "The virtual origin as a first-order correction for the far-field description of laminar jets," Physics of Fluids, vol. 14, no. 6, pp. 1821-1824, 2002.

[7] P. Astin and G. Wilks, "Laminar jet assimilation into non-uniform flows," Proceedings of the Royal Society. London. Series A, vol. 453, no. 1958, pp. 593-606, 1997.

[8] P. Astin and G. Wilks, "The flow of pre-heated, two-dimensional laminar jet issuing into an aligned uniform stream," Zeitschrift für Angewandte Mathematik und Physik, vol. 53, no. 2, pp. 308-322, 2002.

[9] J. Barker and G. Wilks, "Axisymmetric wake and jet solutions in decelerating streams," Zeitschrift für Angewandte Mathematik und Physik, vol. 53, no. 1, pp. 35-47, 2002.

[10] R. S. Brand and F. J. Lahey, "The heated laminar vertical jet," Journal of Fluid Mechanics, vol. 29, pp. 305-315, 1967.

[11] M. Sánchez-Sanz, M. Rosales, and A. L. Sánchez, "The hydrogen laminar jet," International Journal of Hydrogen Energy, vol. 35, no. 8, pp. 3919-3927, 2010.

[12] O. G. Martynenko, V. N. Korovkin, and Y. A. Sokovishin, "The class of self-similar solutions for laminar buoyant jets," International Journal of Heat and Mass Transfer, vol. 32, no. 12, pp. 2297-2307, 1989.

[13] R. Hornbeck, Numerical Marching Techniques for Fluid Flows with Heat Transfer, National Aeronautics and Space Administration, Washington, DC, USA, 1973.

[14] J. C. Tannehill, D. A. Anderson, and R. H. Pletcher, Computational Fluid Mechanics and Heat Transfer, Taylor \& Francis, 2nd edition, 1997. 
[15] M. Noskov, M. Benzi, and M. D. Smooke, "An implicit compact scheme solver for two-dimensional multicomponent flows," Computers and Fluids, vol. 36, no. 2, pp. 376-397, 2007.

[16] W. S. Yu, H. T. Lin, and H. C. Shih, "Rigorous numerical solutions and correlations for twodimensional laminar buoyant jets," International Journal of Heat and Mass Transfer, vol. 35, no. 5, pp. 1131-1141, 1992.

[17] H. B. Aissia, Y. Zaouali, and S. El Golli, “Numerical study of the influence of dynamic and thermal exit conditions on axisymmetric laminar buoyant jet," Numerical Heat Transfer; Part A, vol. 42, no. 4, pp. 427-444, 2002.

[18] A. Ali, N. Kechiche, and H. Ben Aissia, "Prandtl-number effects on vertical buoyant jets in forced and mixed convection regimes," Energy Conversion and Management, vol. 48, no. 5, pp. 1435-1449, 2007.

[19] M. Rosales-Vera and A. Valencia, "A note on Fourier series solution to the plane jet," Far East Journal of Applied Mathematics, vol. 37, no. 1, pp. 103-110, 2009.

[20] A. Kumar and K. S. Yajnik, "Internal separated flows at large Reynolds number," Journal of Fluid Mechanics, vol. 97, no. 1, pp. 27-51, 1980.

[21] A. Plotkin, "Spectral method solutions for some laminar channel flow with separation," in the 20th AIAA Aerospace Sciences Meeting, January 1982.

[22] E. Figueira Da Silva and R. M. Cotta, "Benchmark results for internal forced convection through integral transformation," International Communications in Heat and Mass Transfer, vol. 23, no. 7, pp. 1019-1029, 1996.

[23] I. K. Khabibrakhmanov and D. Summers, “The use of generalized Laguerre polynomials in spectral methods for nonlinear differential equations," Computers $\mathcal{E}$ Mathematics with Applications, vol. 36, no. 2, pp. 65-70, 1998.

[24] M. A. Rosales and R. L. Frederick, "Semi analytic solution to the cartesian graetz problem: results for the entrance region," International Communications in Heat and Mass Transfer, vol. 31, no. 5, pp. 733-740, 2004.

[25] H. Ma, W. Sun, and T. Tang, "Hermite spectral methods with a time-dependent scaling for parabolic equations in unbounded domains," SIAM Journal on Numerical Analysis, vol. 43, no. 1, pp. 58-75, 2005.

[26] M. Rosales and A. Valencia, "A note on solution of Blasius equation by Fourier series," Advances and Applications in Fluid Mechanics, vol. 6, no. 1, pp. 33-38, 2009.

[27] M. Rosales-Vera and A. Valencia, "Solutions of Falkner-Skan equation with heat transfer by Fourier series," International Communications in Heat and Mass Transfer, vol. 37, no. 7, pp. 761-765, 2010.

[28] C. Canuto, M. Y. Hussaini, A. Quarteroni, and T. A. Zang, Spectral Methods: Evolution to Complex Geometries and Applications to Fluid Dynamics, Scientific Computation, Springer, Berlin, Germany, 2007.

[29] R. Moreau, “Jet libre plan, laminaire, d'un fluide incompressible en présence d'un champ magnétique transversal," Comptes Rendus de l'Académie des Sciences, vol. 256, pp. 2294-2296, 1963.

[30] J. Vlachopoulos, "A numerical study of laminar magnetohydrodynamic free jets," Computers $\mathcal{E}$ Fluids, vol. 2, no. 3-4, pp. 261-271, 1974. 


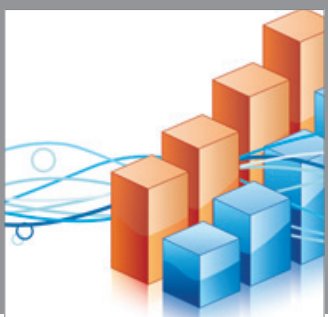

Advances in

Operations Research

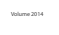

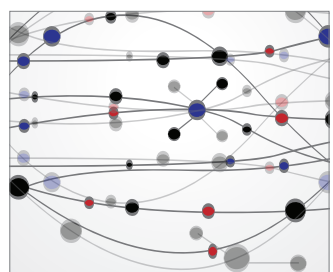

\section{The Scientific} World Journal
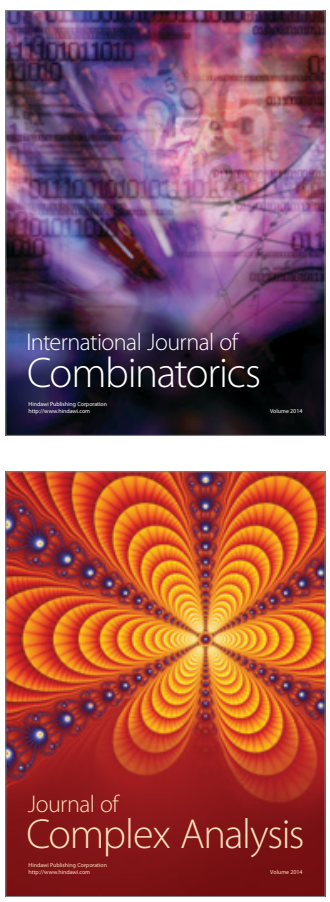

International Journal of

Mathematics and

Mathematical

Sciences
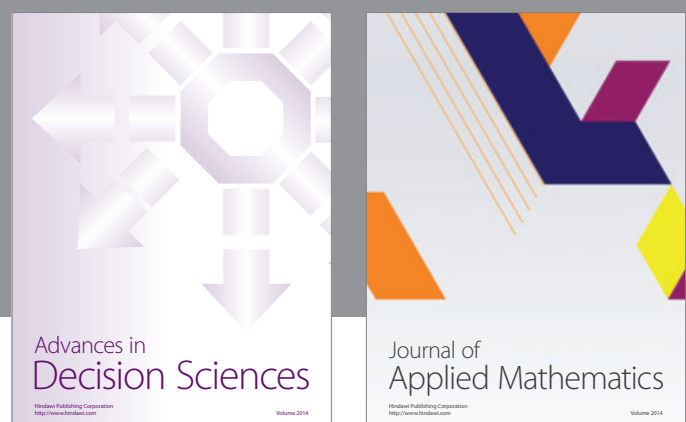

Journal of

Applied Mathematics
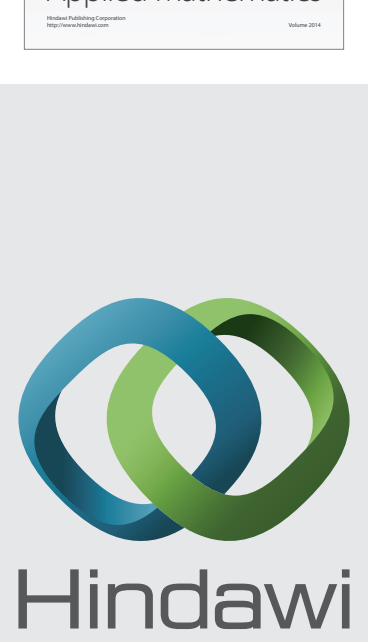

Submit your manuscripts at http://www.hindawi.com
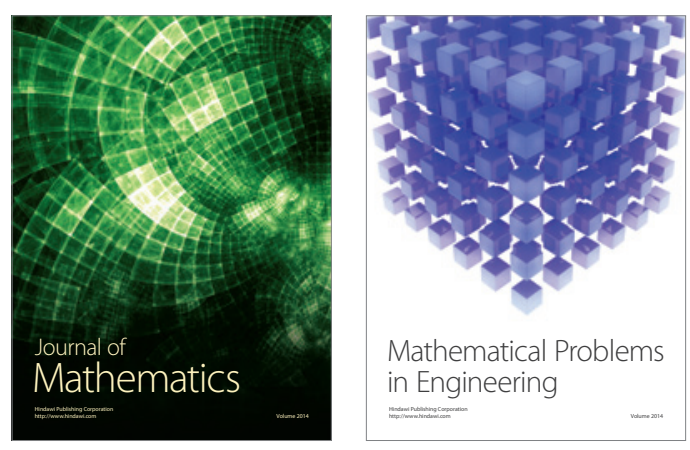

Mathematical Problems in Engineering
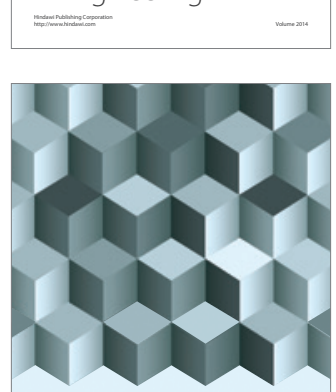

Journal of

Function Spaces
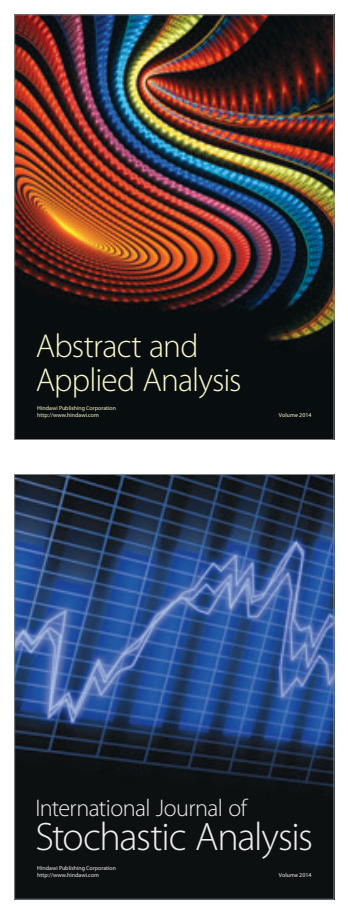

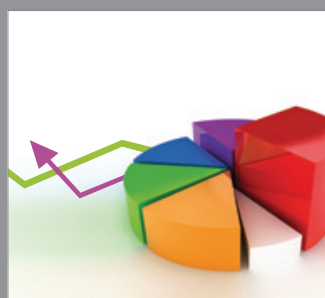

ournal of

Probability and Statistics

Promensencen
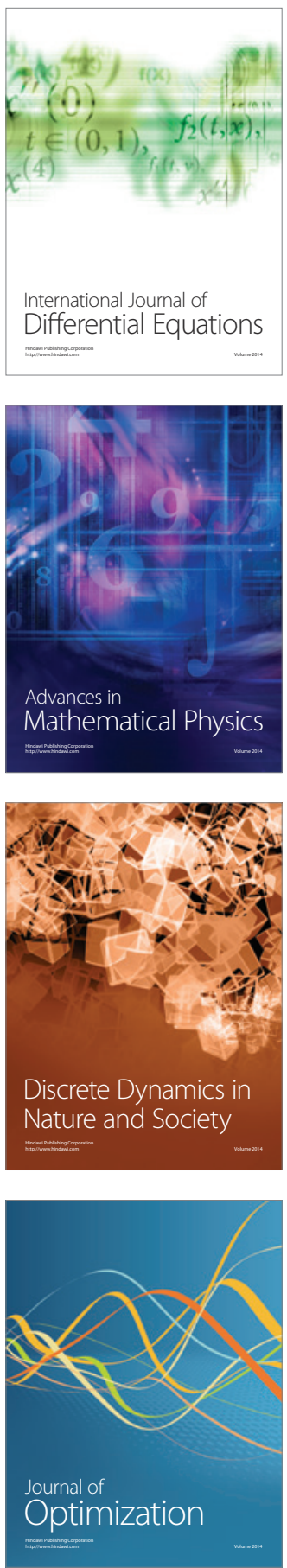